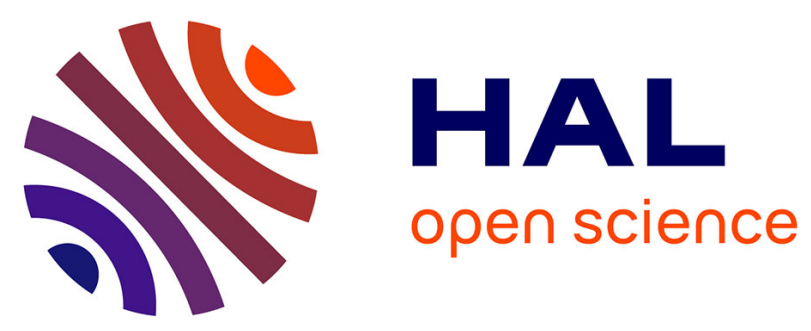

\title{
Numerical study of dynamic relaxation with kinetic damping applied to inflatable fabric structures with extensions for 3D solid element and non-linear behavior
}

Javier Rodriguez Garcia, Gérard Rio, Jean-Marc Cadou, Julien Troufflard

\section{- To cite this version:}

Javier Rodriguez Garcia, Gérard Rio, Jean-Marc Cadou, Julien Troufflard. Numerical study of dynamic relaxation with kinetic damping applied to inflatable fabric structures with extensions for 3D solid element and non-linear behavior. Thin-Walled Structures, 2011, 49 (11), pp.1468-1474. 10.1016/j.tws.2011.07.011 . hal-00715961

\author{
HAL Id: hal-00715961 \\ https://hal.science/hal-00715961
}

Submitted on 2 Jul 2018

HAL is a multi-disciplinary open access archive for the deposit and dissemination of scientific research documents, whether they are published or not. The documents may come from teaching and research institutions in France or abroad, or from public or private research centers.
L'archive ouverte pluridisciplinaire HAL, est destinée au dépôt et à la diffusion de documents scientifiques de niveau recherche, publiés ou non, émanant des établissements d'enseignement et de recherche français ou étrangers, des laboratoires publics ou privés. 


\title{
Numerical study of dynamic relaxation with kinetic damping applied to inflatable fabric structures with extensions for 3D solid element and non-linear behavior
}

\author{
J. Rodriguez*, G. Rio, J.M. Cadou, J. Troufflard

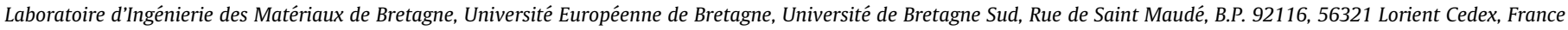

\begin{abstract}
This work mainly deals with the numerical study of inflatable fabric structures. As implicit integration schemes can lead to numerical difficulties such as singular stiffness matrices, explicit schemes are preferred. Since the final objective of this study is to obtain the final shape of a structure, a dynamic relaxation (DR) method is used. These methods allow us to obtain the final and stable shape of the inflatable fabric structures without doing so many time increments, which is the case when using a classical explicit integration method. Han and Lee [5] proposed an extension of the DR method stated by Barnes [13] suitable for triangular elements and elastic behavior. There are two main contributions in this paper. Firstly, we propose a modification of Han and Lee's method, allowing it to be used with any kind of membrane or solid finite elements and any reversible behavior. Secondly, we propose to rewrite the expression initially introduced by Barnes. Furthermore, these proposals are adapted for incremental loadings, allowing this way to obtain the pseudo-equilibriums of the intermediate phases. Numerical examples from academic problems (rectangular and circular membranes) show the efficiency and the reliability of proposed methods, with linear elasticity behavior, and also with a non-linear incremental behavior and finite deformation states.
\end{abstract}

\section{Introduction}

The simulation by the FE method of inflatable fabric structures, when a pressure load is applied and an implicit scheme is used, can lead to severe instabilities due to the lack of stiffness in the fabric. For instance, in certain cases the basic Newton-Raphson algorithm cannot achieve a final convergence due to the swapping between several stable states. Explicit time schemes overcome this difficulty, but they need a huge number of time steps to obtain a realistic stable final shape. This occurs when using natural damping.

This is an usual issue in civil engineering (some examples are: geotechnical problems [1], prestressed coated fabric membranes [2], architectural structures [3], and space inflatable structures [4]), and there have been several solutions proposed [5-9] by using dynamic relaxation methods.

The classic form of the dynamic relaxation (see for example [10] or [11]) mainly is to use an artificial viscosity to damp the movement, and to search for the critical damping value (see [12] for a comprehensive review). However, among the existing dynamic relaxation methods, we are interested in a different method; the

\footnotetext{
* Corresponding author. Tel.: +33 643122334; fax: +33 297874572.

E-mail address: javirguezg@gmail.com (J. Rodriguez).
}

one proposed by Barnes [13]. It has been initially applied to the calculation of prestressed cable structures and further extended by Han and Lee [5] to be used with triangular elements and a linear elastic behavior. This method combines a kinetic damping (resetting the speed to zero at each kinetic energy peak), often used in form-finding, and an optimization of the mass matrix (proposed by Han and Lee).

One application of the method is thin fabric structures loaded by pressure, which are notably unstable during loading due to the lack of flexion stiffness. The static final form does not have to depend on the inertial forces that act during the transient evolution. Considering this, the right value of the mass is supposed to have no influence on its static final form. In order to quickly reach the stable deformed state, we must first adapt the mass matrix and then use kinetic damping. Kinetic damping has been successfully employed by several authors (one example is [15]). A correct choice of the mass matrix leads to an optimal convergence of the dynamic relaxation method.

In this paper, we will present two main formulations. Firstly, we propose an extension of that Barnes-Han-Lee method. Secondly, we propose a general expression based on the works of Barnes for the mass matrix calculus. The basis of this second expression has already been proposed in previous papers (see Underwood [16] or Barnes [13]), but to our knowledge, no systematic studies have 
been done concerning its applications for simulation of the inflation of unstable structures. Both formulations can be used with any reversible behavior, any type of membrane element and also solid elements. We show, with numerical examples, their correct operation even when dealing with complex mesh shapes or 3D elements. Our methods aim to find one solution when one or more solutions exist (there can be several stable final shapes).

The reminder of this paper is broken into three main parts. In Section 2, we first explain the dynamic relaxation method that our work is based on. We then present two methods for the formulation of the mass matrix. We propose an initial method based on the formulation of Han and Lee and also propose an alternate method based on a different formulation of the mass matrix. In Section 3, we show several numerical case studies and results of application of our proposals after implementing them in the software Herezh++ [17]. Finally, in Section 4, we briefly discuss our conclusions.

\section{Dynamic relaxation method}

\subsection{Presentation of the method}

The problem that we need to solve, after discretization by $\mathrm{FE}$, is

$[M] \ddot{X}+R(X, \dot{X})=0$

where $[M]$ is the diagonal mass matrix; $X, \dot{X}, \ddot{X}$ are respectively the position, velocity and acceleration of the nodes and $R(X, \dot{X})$ is the residual of internal and external forces in function of the position and velocity of nodes. The term $[M] \ddot{X}$ represents the generalized expression of the acceleration forces.

The method used to solve the problem in time is based on the explicit centered finite differences method (CFD).

When a structure is steady and immobile, its kinetic energy is equal to zero and acceleration forces are null. In order to quickly reach this state, a kinetic damping is used. The kinetic damping method consists in resetting velocity to zero at each kinetic energy peak. This simple procedure has generally been found to be stable and allowing a fast convergence [13]. When there is no external energy acting, there is just internal elastic energy to evolve toward the steady-state position.

The calculation of equilibrium is always made in the final configuration, using the Cauchy's stress tensor.

In the case of a Hooke's elastic law of behavior, Cauchy's tensor is associated with Almansi's deformation measure. The deformation is calculated from the variation of the coordinates of the metric tensor associated to the material coordinates of the point in the reference element (a comprehensive example of this calculus has been presented in [21]). Then, the stress tensor is obtained by means of the formulae

Spheric part : $\frac{\operatorname{tr}(\sigma)}{3}=K \operatorname{tr}(\varepsilon)$

Deviatoric part : $\operatorname{dev}(\sigma)=2 G \operatorname{dev}(\varepsilon)$

In the case of the more complex material behavior: Hart-Smith, that we use in one of our numerical examples, the tensor of left Cauchy-Green B is determined, using again the coordinates of the metric tensor. Its invariants are then used to define the potential and its different variations, needed for the calculation of Cauchy's stress tensor [21].

For both of these behaviors, only the initial and final states are taken into account.

Since acceleration forces depend on the mass (see equation to solve above), the dynamic relaxation method proposed by Barnes [13] uses an arbitrary mass term in order to improve the kinetic damping while keeping the numerical stability. In the previous studies, Barnes proposes a lumped mass matrix where the elements $m_{i}$ in the diagonal are

$\left[m_{i}\right]=\lambda \frac{\Delta t^{2}}{2}\left[k_{i}\right]$

where $m_{i}$ is the mass matrix at node $i$; $k_{i}$ represents the diagonal component of the stiffness matrix in the principal direction; $\Delta t$ is arbitrarily chosen as 1 ; and $\lambda$ is a convergence parameter which is constant for the whole structure. The optimum mass matrix is calculated by adjusting the parameter $\lambda$.

In the shape-finding process of membrane structures, due to the large variations of the structures, Barnes [14] proposed to choose the largest stiffness term for the calculation of mass term:

$m_{x i}=m_{y i}=m_{z i}=\lambda \frac{\Delta t^{2}}{2} k_{\text {imax }}$

and Han and Lee [5] stated, for CST (constant stress triangle) elements, that the stress $k_{i}$ at node $i$ with $m$ members can be approximated as

$k_{\text {imax }}=\sum_{e} \frac{h}{4 S_{0}^{e}}\left(\frac{E}{1-v^{2}}+\sigma_{x}+\sigma_{y}+\sigma_{x y}\right)$

where $h$ is the thickness of the element $e$; $S_{0}^{e}$ is the initial surface of the element $e$; and $\sigma_{x}, \sigma_{y}, \sigma_{x y}$ are the components of the stress tensor in an orthonormal basis associated to the surface element. $E$ and $v$ are the coefficients of the isotropic elastic Hooke's behavior law.

The time step is arbitrary, and for simplicity, its value in the formula (4) has been chosen as 1 . The consequence is that this time step does not appear directly in the time-advance algorithm, but it does in the loading, boundary conditions and material's behavior.

In Ref. [18], the authors propose to suppress the surface term $S_{0}^{e}$ in order to obtain mass dimensions in Eq. (4). They show, particularly, that in this case the optimal value of the coefficient $\lambda$ is more stable, what is advantageous when it has to be defined. The expression becomes then

$k_{\text {imax }}=\sum_{e} \frac{h}{4}\left(\frac{E}{1-v^{2}}+\sigma_{x}+\sigma_{y}+\sigma_{x y}\right)$

In this work, we propose two ways to generalize the previous approaches, which we define in the two following subsections.

\subsection{Proposal 1: extension of the formulation of Barnes-Han-Lee}

Here we propose an extension of the previous formulation, on the one side to other type of elements and on the other side to other material's behavior. The aim is therefore to study the feasibility of this extension. Let us consider the following expression, which would replace Han-Lee's [5]:

$k_{\text {imax }}=\sum_{e} \frac{l_{e}}{4}\left(\alpha K+\beta \mu+\gamma \frac{I_{\sigma}}{3}+\frac{\theta}{2} \sigma_{\text {mises }}\right)$

Looking at the expression (6), the term $E /\left(1-v^{2}\right)$ can be considered as controlling the shape changing or the element volume changing. It could be replaced by a linear combination of the average compressibility modulus $K$ and shear modulus $\mu$, available for all elastic and hyperelastic laws: $\alpha K+\beta \mu$.

Initially, parameters $\alpha$ and $\beta$ can be chosen as $\alpha=\beta=1$, what leads to a magnitude almost equal to the initial formulae's one (with the condition of a not very high compressibility). For example, if $v=0.3$, we get: $\alpha K+\beta v \approx 1.21 E$, while with Han-Lee's formulae (6) we obtain: $E /\left(1-v^{2}\right) \approx 1.1 E$.

Concerning the second part of the Eq. (6) proposed by Han-Lee, the term $\sigma_{x}+\sigma_{y}+\sigma_{x y}$ can be considered as representing the stress state in the material (cumulating the spheric and 
deviatoric parts). For our proposal, and in order to extent the use of the formulae to other geometries than triangular elements, we replace this term by an invariants' combination: $\gamma\left(I_{\sigma} / 3\right)+(\theta / 2) \sigma_{\text {mises }}$, where $I_{\sigma}=\sigma_{k}^{k}$ is the trace of the Cauchy stress tensor and $\sigma_{\text {mises }}$ is the Mises stress. They represent respectively the intensity of the spherical and the deviatoric parts of the stress tensor. These two quantities are tensor invariants so they could be calculated for any type of element.

Therefore, the coefficient $1 / 3$ is added so $-I_{\sigma} / 3$ represents the spherical pressure, and the coefficient 0.5 is added so $0.5 \sigma_{\text {mises }}$ represents the amount of shear.

The parameters $\alpha, \beta, \gamma$ and $\theta$ in the expression (8) permit to control the influence of each entity. And finally, $l_{e}$ represents a geometrical characteristic length, suitable for 2D elements (thickness) and for 3D elements (cubic root of the volume).

Notice the presence of the stress terms, what implies that the mass matrix has to be updated all along the calculation.

\subsection{Proposal 2: second formulation for the mass matrix}

The second proposal refers to the theoretical elements proposed by the early work of Underwood [16] by using the theorem of Gerschgorin which permits to obtain an upper bound to the eigenvalue " $\mathrm{i}$ " of the stiffness matrix " $\mathrm{K}$ " of the system:

$\rho_{i} \leq \sum_{j}\left|K_{i j}\right|$

The mass matrix is then built to satisfy the stability condition with a unitary time step

$m_{i}=\frac{\lambda}{2} M A X_{k=1}^{3}\left(\rho_{3(i-1)+k}\right)$

Unlike the physical masses, we can expect a variation of the mass matrix built this way during the calculation. Given that on the one side we choose the maximum value over the three axes (loop over $\mathrm{k}$ in (10)) and on the other side the stiffness of the initial material behavior is generally more important than during deformation, it has been proved in our simulations that the mass matrix calculated at the beginning was enough to "guide" the whole simulation, i.e. the update of the mass matrix along the calculation of our simulations did not provoke any time gainings.

The method presents as a disadvantage that it needs at least the calculation of one stiffness matrix, what implies the need of being able to calculate the tangent behavior. Generally, at the beginning of the loading process, the evolution is mainly elastic, so a priori the stiffness belonging to the tangent behavior should be enough if we consider that the material tends to soften.

In order to make the calculations converge as fast as possible, the idea is to be as close as possible to the critical time step. However, being too close to the critical time step can eventually provoke instabilities and divergence. In the dynamic relaxation method formulae, the time step $\Delta t$ is usually arbitrarily fixed to 1 . However, the presence of the parameter $\lambda$ can be considered as a factor of this fixed time step. Its optimum value cannot be determined a priori (we observed that it depends on the mesh geometry, elements size, etc.). However, the relation (9) and the formulae (2.4) of Ref. [16] lead to a minimum theoretical value of $\lambda$ for the second proposal: $\lambda \geq 0.5$.

The range of values to find the optimum value of $\lambda$ is different for each one of our proposals, being this range much smaller in the case of the second proposal (around 0.5-0.7 for the proposal 2 vs. 5-15 for the proposal 1 ).

\subsection{Incremental scheme and convergence criterion}

In the case of an incremental law of behavior, a priori not totally reversible, when the loading leads to big deformation-stress final states, the final-form-finding procedure in one step is not correct anymore. The final state depends indeed on the loading path which in the case of DR can be very different to the real path. A solution is to use an incremental loading procedure. Assuming that increments are small enough, the procedure then guarantees a succession of points of static physical equilibrium that allows to be close to the real response of the structure during the loading.

The convergence criterion in the steady state must comply with two points. In one part, the structure must be in mechanical equilibrium, what we represent as a norm of the residual of the static generalized forces - internal and external - inferior to an instruction's value. In other part, in the case where the kinematic boundary conditions block the global solid movements, we impose the final kinetic energy to be also inferior to an instruction value, what also guarantees the equilibrium in the case where the generated stresses are very low. In the practical, these conditions are applied under a relative form according to

$\operatorname{Max}\left(\frac{\| \text { Residual } \|_{\infty}}{\| \text { Reactions } \|_{\infty}}, \frac{\text { Kinetic energy }}{\text { Internal energy }}\right) \leq \varepsilon$

where $\varepsilon$ is the convergence criterion (the mentioned instruction value).

\section{Numerical case studies}

In this section, we will perform numerical case studies on the formulae (8) and (10). We use the $\mathrm{C}++$ academic finite elements software Herezh++ [17], and for the meshing and postprocessing, we use the software Gmsh [19].

The numerical case studies in the section are described below:

- Firstly, we show how we can adapt the parameters of the first of our proposed formulae to obtain an equivalent calculation to Han and Lee's formulation. We also compare it to our second formulae using the classical test of inflation of a rectangular cushion.

- Based on the same numerical test, we show that both of our proposed formulae work with complex meshes, with different geometries, with different element types and different interpolations.

- We demonstrate our proposed formulae working with meshes composed of 3D elements, using an inflate test of a rectangular cushion and a traction test of a partially perforated plate.

- Finally, we explore how the formulae works when using a complex law of behavior.

\subsection{Equivalence between the formulation of Han and Lee and our proposals}

The objective in this section is to verify the equivalent calculation between the method proposed by Hann and Lee, (6), modified by Troufflard [18] and our formulae (8) and (10). Notice that considering the term $\sigma_{x y}$ in the formulae (6) is problematic, because it depends on the coordinates on which it is calculated. In our work, in order to suppress this dependance, we propose to be situated in a stress eigenvector frame, which leads, considering the plain stress hypothesis, to $\sigma_{x y}=0, \sigma_{x}+\sigma_{y}=\operatorname{tr}(\sigma)$ and $\gamma=3$ in (6).

The solution permitting to determine the terms $\alpha$ and $\beta$ is not unique. We keep

$\alpha=\frac{3(1-2 v)}{2\left(1-v^{2}\right)}, \quad \beta=\frac{1}{1-v} \quad$ being $K=\frac{E}{3(1-2 v)}, \quad \mu=\frac{E}{2(1+v)}$ 
In this case, relations (6) and (8) are identical, what shows that our first proposal includes the former Han-Lee's proposal.

The calculation is carried out in linear elasticity, $E=125 \mathrm{MPa}$ and $v=0.41$, which are coherent with the parameters of behavior of a usual thin fabric. Concerning the expression (10), we use for this example a value of $\lambda=0.6$ (we will discuss about this parameter afterwards).

The first numerical test is a classical one, which has already been studied, for example, in Ref. [6], the inflation of a rectangular shaped cushion. It consists in two membranes joined at their periphery, with dimensions $500 \mathrm{~mm} \times 500 \mathrm{~mm} \times 0.27 \mathrm{~mm}$. Due to the symmetries, just $1 / 8$ of the cushion is studied. The cushion is loaded with an instantaneous internal pressure of $0.015 \mathrm{MPa}$. The mesh is constituted of a ruled triangular division, $25 \times 25 \rightarrow 625$ elements.

Finally, the convergence criterion (11) is set to: $\varepsilon=1 . e-3$.

The evolution of kinetic energy in function of the iteration number, Fig. 1, shows clearly the kinetic damping points (i.e. speeds reset to 0 ). Globally, the evolution for both proposals is very close. Entering into detail, notice that the first maximum of kinetic energy occurs a bit later in the case of the second proposal, but on the other hand, the decrease's gradient seems to be bigger for this second proposal. We can also notice the regular diminution of the norm of static residual, quite similar for both methods, with an important diminution at each kinetic damp.

\subsection{Membranes: complex meshes}

The aim of the second application is to validate our two proposals in the case of more complex meshes. So we study 2D meshes, with triangular and quadrangular elements, and with linear and quadratic interpolations. Also, two different qualities of mesh are considered: a grid of $25 \times 25$ elements and another one of $50 \times 50$. The geometry and the material behavior are identical to the first application, in the previous section. For the first proposal, we use the parameters $\alpha=0.9022557, \beta=0.9022557$, $\gamma=1, \theta=1$.

For the different tested samples, we use the notation indicated in Table 1. Thus, as an example, the notation RL1 means: test made with a mesh with $25 \times 25$ rectangular elements, and using linear interpolation.

The Fig. 2 shows an example of inflated membrane. There, we can observe that the method permits also to capture eventual wrinkles, local instabilities which can appear during the inflation. We would like to remark that it is not the aim of this work to obtain the best precision for the wrinkles. It is evident that, the smaller the mesh is, the more we gain in wrinkles' precision. But as told, the aim of this work is to obtain one among all the possible solutions for the steady inflated state; we cannot assure that the obtained wrinkles would have the same shape and would be placed in exactly the same position than in reality. To assure that, further studies must be made.

For each geometry, the Table 2 shows the obtained results with an optimum $\lambda$. Calculations are made in an Apple computer (Processor: $2 \times 2.93 \mathrm{GHz}$ Quad-Core Intel Xeon, Memory: 16 Go $1066 \mathrm{MHz}$ DDR3) with just one processor.

Firstly, it can be observed that convergence is reached in all the cases. Particularly, the quadratic interpolation does not induce a particular difficulty.

Table 1

Notation.

\begin{tabular}{ll}
\hline $\begin{array}{l}\text { T: triangular } \\
\text { elements }\end{array}$ & $\mathbf{R}$ : rectangular elements \\
$\begin{array}{ll}\text { L: linear } \\
\text { interpolation }\end{array}$ & Q: quadratic \\
1: mesh of $25 \times 25$ & 2: mesh of $50 \times 50$
\end{tabular}
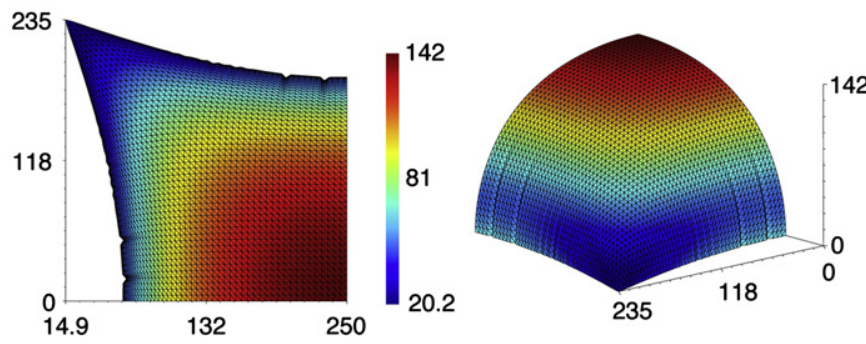

Fig. 2. Inflated squared cushion: representation of $1 / 8$ of the cushion, displacement isovalues.

Table 2

Inflation of $1 / 8$ of cushion in just one loading step, for different meshes.

\begin{tabular}{|c|c|c|c|c|c|c|}
\hline \multirow[t]{2}{*}{ Mesh } & \multicolumn{3}{|c|}{ Proposal 1} & \multicolumn{3}{|c|}{ Proposal 2} \\
\hline & $\lambda_{o p t}$ & Iterations & Time (s) & $\lambda_{o p t}$ & Iterations & Time (s) \\
\hline TL1 (2028 dof) & 10 & 546 & 14.1 & 0.6 & 565 & 13.8 \\
\hline TL2 (7803 dof) & 10 & 923 & 101.5 & 0.7 & 1081 & 111.8 \\
\hline TQ1 (7803 dof) & 13 & 1128 & 118.4 & 0.6 & 1185 & 119.8 \\
\hline TQ2 (30 603 dof) & 14 & 2158 & 943.3 & 0.7 & 2358 & 970.1 \\
\hline RL1 (2028 dof) & 6 & 422 & 23.7 & 0.5 & 423 & 22.6 \\
\hline RL2 (7803 dof) & 6 & 671 & 150.4 & 0.6 & 841 & 183.9 \\
\hline RQ1 (7803 dof) & 10 & 1015 & 159.8 & 0.5 & 970 & 148.8 \\
\hline RQ2 (30 603 dof) & 9 & 1688 & 1085.6 & 0.5 & 1889 & 1552.1 \\
\hline
\end{tabular}

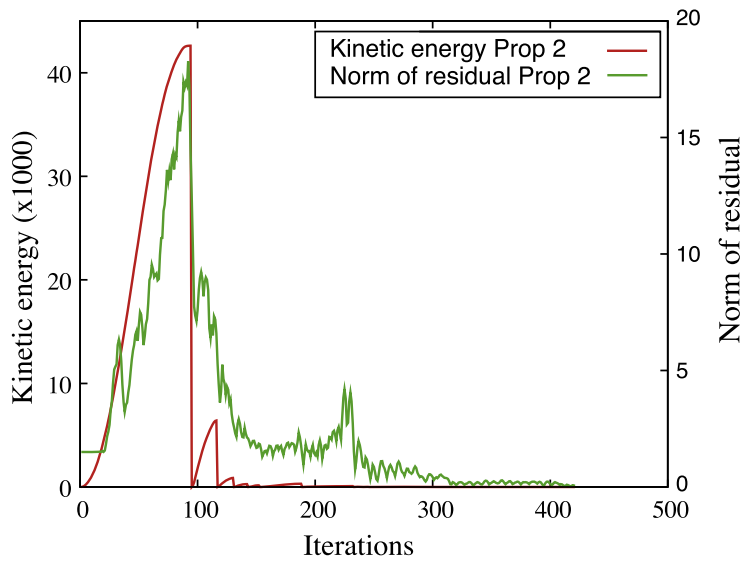

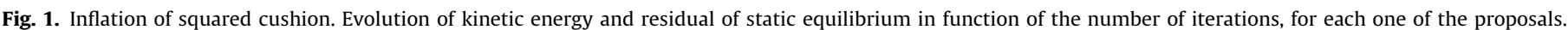


The number of needed iterations is slightly higher with the second proposal, but the associated calculation times stay however smaller or equivalent, due to the fact that the stiffness matrix is not recalculated at each iteration, unlike the proposal 1.

The range of variation of the parameter $\lambda$, even if it is smaller than in the original method of Barnes-Han-Lee, (6), it is still quite large for the proposal 1 . This point is important, because it imposes preliminary tests in the case of a full new mesh in order to determine the optimum value for $\lambda$. In the case of the proposal 2 , a value of 0.6 or 0.7 ensures a convergence near the optimal one in all the studied cases.

The increasing number of iterations seems to be proportional to the square root of the number of dof, i.e. the quality of the mesh.

\subsection{Circular mesh}

The third application concerns the inflation of a circular cushion, with a diameter or $400 \mathrm{~mm}$, where the mesh, Fig. 3, includes both triangular and quadrilateral linear elements. The other material, geometric, etc., characteristics are identical to the squared cushion's ones, and also the methods.

Table 3 shows that the number of necessary iterations for convergence is coherent with those obtained for squared geometries. The mix of elements does not seem to alter the convergence. The proposal 2 is here more interesting, because even with the same previously used value of $\lambda=0.6$, which is not the optimum, we obtain a very good convergence.

We can also observe the presence of wrinkles in the solution. Analogously to a classic explicit dynamic scheme, considering these instabilities does not seem to create a problem. However, the process does not allow to control the choice of the bifurcated solution.

\subsection{Meshes with $3 D$ elements}

We consider now the case of 3D elements. Two different types of simulations are studied: a squared plate under a transversal

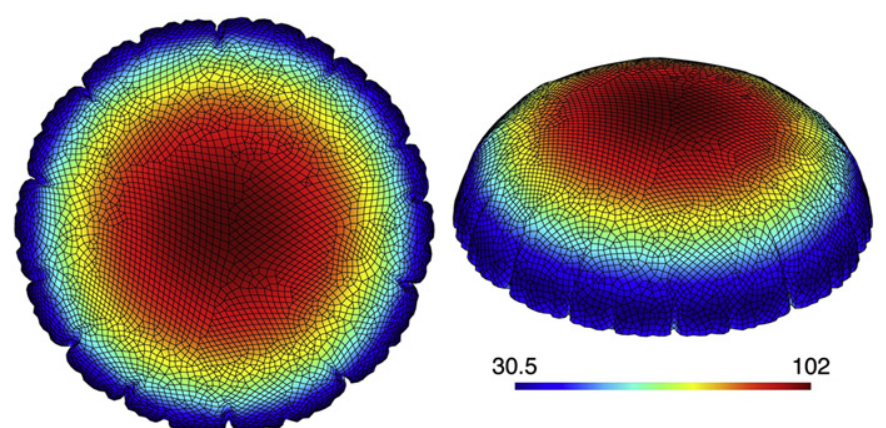

Fig. 3. Inflated circular cushion: displacement isovalues.

Table 3

Inflation of a half of a circular cushion, with a mix of linear triangular and quadrangular elements.

\begin{tabular}{|c|c|c|c|c|c|c|}
\hline \multirow[t]{2}{*}{ Mesh } & \multicolumn{3}{|c|}{ Proposal 1} & \multicolumn{3}{|c|}{ Proposal 2} \\
\hline & $\lambda_{o p t}$ & Iterations & Time (s) & & Iterations & Time (s) \\
\hline Circular & 10 & 2096 & 1068.3 & $\lambda_{o p t}=0.4$ & 1322 & 616.745 \\
\hline 17856 dof & & & & $\lambda=0.6$ & 1703 & 792.6 \\
\hline
\end{tabular}

load (pressure), and a partially perforated plate (just called perforated plate in the rest of the paper for simplicity) under a load in the plane. The thickness of both plates is $5 \mathrm{~mm}$.

The first case represents the version in 3D elements of the inflation previously studied, with also, in this case, a certain rigidity to flexion of the plate. In fact, to obtain similar deformations, we use now a pressure of $0.08 \mathrm{MPa}$. While in the first case the deformation-stress fields are quite homogeneous in the plane (not in the thickness), and the displacements are important, in the second case, the presence of the partial hole originates an important field gradient in the plane, and displacements are comparatively smaller. Both classic interpolations, linear and quadratic are used, and also two types of element: hexahedral and pentahedral. Both proposals give similar results, but just the proposal 2 is shown here, with $\lambda=0.7$.

Table 4 shows that in all cases the calculation converges, even if it is for large deformations in the plane (Fig. 5) or if it is for large transversal displacements including flexion (Fig. 4). In the case of quadratic hexahedra, the studied case consists in a complete interpolation with 27 nodes and 27 integration points. A priori, this choice permits to avoid the locking in flexion, and despite the fact of having just one element in the thickness, the behavior in flexion is a priori correctly approximated. Obviously, this is not the case for the mesh of linear hexahedra, where it would be necessary to correctly approach the behavior in flexion, to include a higher number of elements in thickness (just one used here), and at least a selective integration to avoid the locking. However, we present both types of interpolation to show, on the one side, that the algorithm converges in both cases, and on the other part, that the calculation times are coherent between them and with the previously obtained results.

For the case of the 3D inflation, considering the ratio of dof between the two meshes (linear and quadratic) is 5.46, and the ratio of integration points is $3.38(=27 / 8)$, we obtain a global ratio of around $5.46 \times 3.38=18.45$. If we compare this ratio with the ratio of calculation times, 24.7 , we can observe that, even if they are not equal, we are in the same order of magnitude.

Concerning the traction tests on the perforated plate, the elements are pentahedra and the global ratio between dof and between quadratic and linear pentahedra's integration points is

Table 4

Tests with 3D elements: meshes and results.

\begin{tabular}{llrrrr}
\hline Case & Element type & Elements & Dof & Iterations & Time (s) \\
\hline Inflation & Linear hexahedra & 100 & 726 & 914 & 21.8 \\
Inflation & Quadr. hexahedra (27 pt) & 100 & 3969 & 2687 & 520.2 \\
Traction & Linear pentahedra & 2239 & 7200 & 1550 & 177.2 \\
Traction & Quadr. pentahedra (6 pt) & 2239 & 31428 & 4140 & 2410 \\
\hline
\end{tabular}

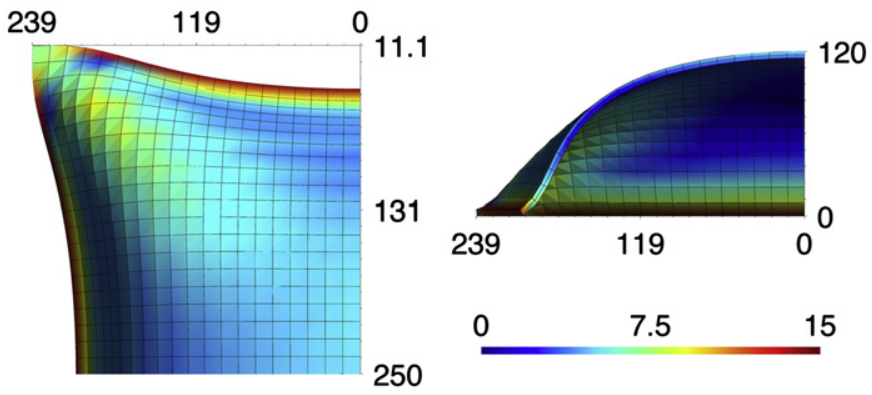

Fig. 4. $1 / 4$ of 3D plate under pressure, result in one step: isovalues of Mises stress (in $\mathrm{MPa}$ ). 
around 13 ( 4.37 for the dof and 3 for the integration points), while the ratio of calculation times is around 13.5. Thus, here also we observe coherent ratios.

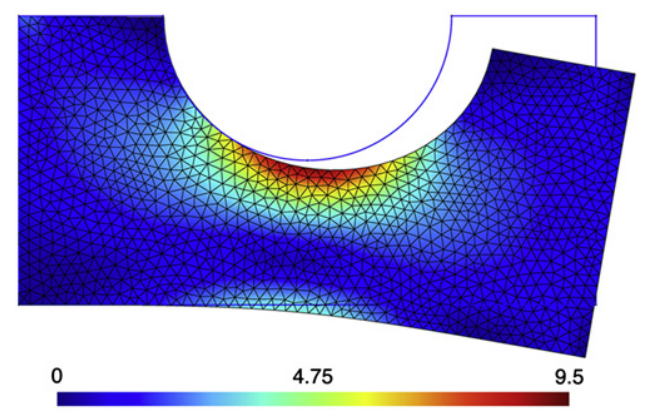

Fig. 5. Perforated plate, result in one step: Mises stress isovalues.

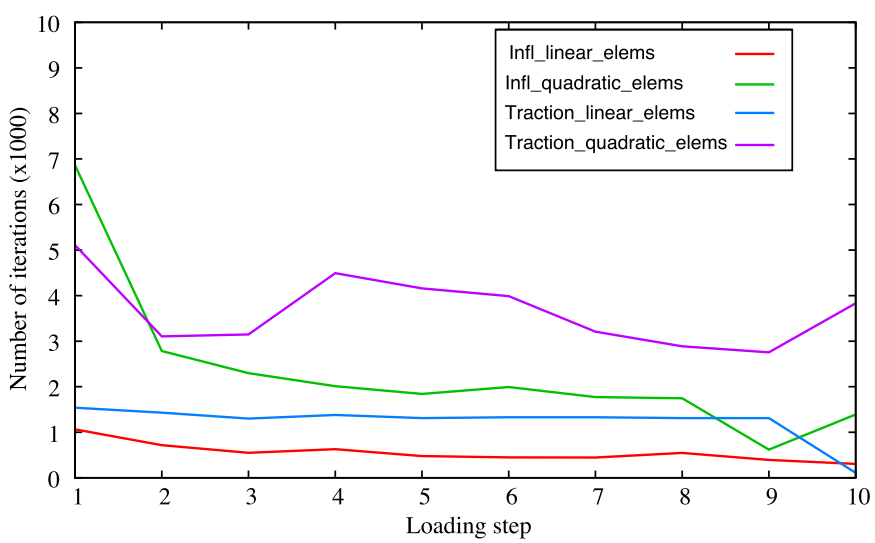

Fig. 6. Number of iterations vs loading step, for the second proposal, and $\lambda=0.7$.

\subsection{Incremental calculations}

In order to be able to use the method in the case of an incremental law of behavior, we introduced an incremental version of the proposals 1 and 2 . Actually, the dynamic relaxation method is used here to find the steady state at the end of each loading step. The method is thus analogous to a classic iterative one, with the difference that it does not need the determination of a tangent evolution; but in return it needs a larger number of iterations.

The different types of simulation 2D and 3D previously presented are studied considering 10 loading steps (increments). As an example, we present results for the proposal 2 . We observe that the method works out for all kind of elements. Fig. 6 presents the evolution of the number of iterations versus the number of increment (loading step). We observe coherent numbers with the one-step calculation. Notice that the number of iterations - quite constant - is less stable with the quadratic than with the linear elements.

Figs. 7 and 8 present the different steps of loading constituting the result of the intermediate pseudo-steady states resulting of the multi-step loading (to improve the clarity of the figures, not all the increments are shown).

\subsection{Complex law of behavior}

The last part of the study is exploratory. It consists in observing the influence of a complex law of behavior, preferably, incremental. For that, we consider the inflation of a squared membrane, meshed with 3D quadratic hexahedral elements. The geometric dimensions are $250 \mathrm{~mm} \times 250 \mathrm{~mm} \times 6 \mathrm{~mm}$, the mesh is constituted of a grid of $10 \times 10 \times 1$ and the used $\lambda$ is 3 (bigger than before, to be sure to overcome nonlinearities). The loading is quasi-static, so the speed effects are negligible. The material is considered an elastomer Vitton where the law is modeled by

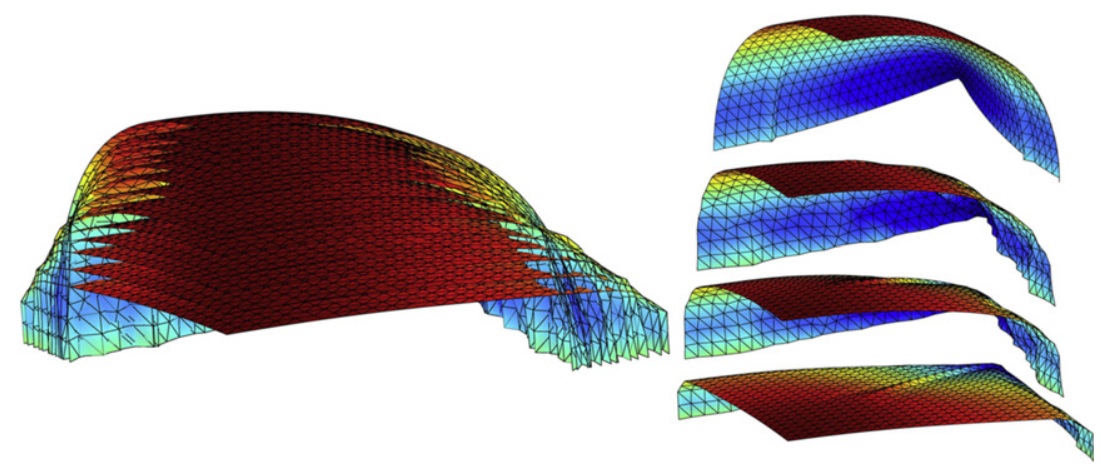

Fig. 7. Incremental inflation of a cushion, representation of $1 / 8$ of the cushion, $2 \mathrm{D}$ mesh.
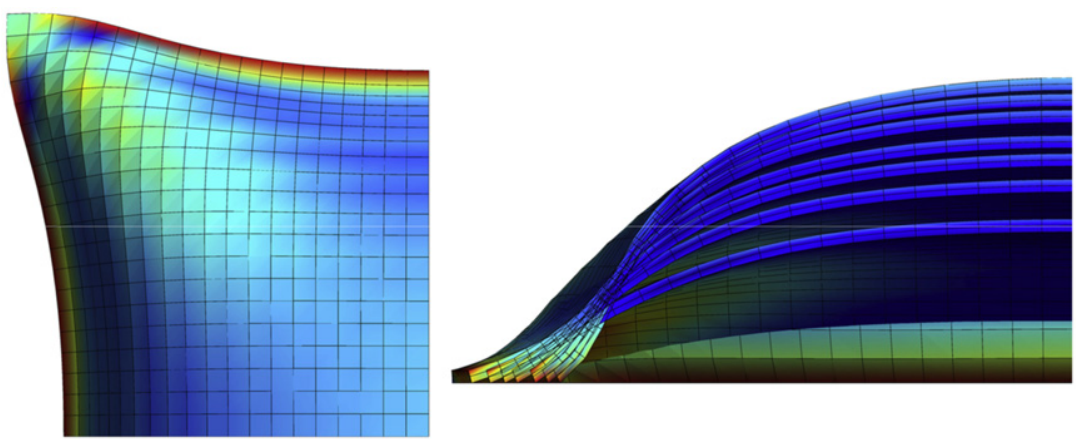

Fig. 8. Incremental inflation of a cushion, representation of $1 / 8$ of the cushion, 3D mesh. 
Table 5

Inflation of an elastomeric plate: needed iterations per loading step.

\begin{tabular}{lllllll}
\hline Mesh & $\lambda$ & Inc 1 & Inc 2 & Inc 3 & Inc 4 & Inc 5 \\
\hline 3969 dof & 3 & 32020 & 8470 & 6540 & 4730 & 2830 \\
& & Inc 6 & Inc 7 & Inc 8 & Inc 9 & Inc 10 \\
& 2910 & 970 & 2590 & 910 & 690 \\
\hline
\end{tabular}

assembling an additive hyperelastic stress and a stress hysteresis. For more details of the law, see [20]. Just notice that the hyperelastic part is based on the model of Hart-Smith: $K=2700 \mathrm{MPa}$ and the deviatoric part coefficients: $C 1=0.067$, $C 2=0.402, C 3=3.05$. The hysteretical behavior is purely deviatoric and incremental and can be seen as an infinite and continuous assembly of couples: a spring and a frictional element in parallel. During a shear test, the slope at the origin is $\tau / \gamma=4.59 \mathrm{MPa}$ and the saturation stress is $\tau_{\max }=0.27 \mathrm{MPa}$.

The calculation converges despite the complex behavior. We observe in Table 5 a number of iterations much higher for the first increment, and then a big regular decreasing of the number of iterations, in opposition to the case of linear elasticity. The reason is that the weak initial stiffness of the material leads to a very big displacement at the first increment. Then, the material rigidifies and the displacements per increment decrease importantly. The observed evolution of the number of necessary iterations in function of the loading step is therefore logical.

\section{Conclusions and discussion}

We presented two proposed formulae to extend Barnes-HanLee's dynamic relaxation method with kinetic damping. BarnesHan-Lee's method was limited to the particular case of linear triangular elements and elastic behavior. Our proposed formulae allow for applications beyond the original limitations. This is our main contribution.

Furthermore, we have numerically demonstrated several other advantages of our formulae. We showed our proposals are effective for 2D and 3D elements, with linear and quadratic interpolation. We showed the formulae are compatible with an incremental formulation, which minimizes the influence of the loading path. Our exploratory work showed that the second proposal works with a complex incremental law of behavior.

We present dynamic relaxation with kinetic damping, using the incremental formulation, as an useful alternative to the classic Newton's method in the cases where instabilities are found.

This work covered structural instabilities. In future work, the study will continue with material instabilities.

\section{Acknowledgments}

This work is financed by Région Bretagne, and in collaboration with the Navimo Society. We thank both of them for their support and collaboration that made it possible.

\section{References}

[1] Dang HK, Meguid MA. Evaluating the performance of an explicit dynamic relaxation technique in analyzing non-linear geotechnical engineering problems. Computers and Geotechnics 2009;37:125-31.

[2] Douthe C, Baverel O, Caron JF. Form-finding of a grid shell in composite materials. Journal of the International Association for Shell and Spatial Structures: IASS 2006;47(150).

[3] Lewis WJ. Tension Structures, Form and Behavior. T. Telford; 2003.

[4] Thomas J, Wielgosz C. Deflections of highly inflated fabric tubes. Thin-Walled Structures 2004;42:1049-66.

[5] Han SE, Lee KS. A study of the stabilizing process of unstable structures by dynamic relaxation method. Computers and Structures 2003;81:1677-88.

[6] Wu TY, Ting EC. Large deflection analysis of 3D membrane structures by a 4-node quadrilateral intrinsic element. Thin-Walled Structures 2008;46: 261-75.

[7] Ramesh G, Krishnamoorthy C. Geometrically non-linear analysis of plates an shallow shells by dynamic relaxation. Computer Methods in Applied Mechanics and Engineering 1995;123:15-23.

[8] Russell C. Deployment simulations of inflatable tensegrity structures. International Journal of Space Structures 2008;23:63-77.

[9] Wood R. A simple technique for controlling element distortion in dynamic relaxation form-finding of tension membranes. Computers and Structures 2003;80:2115-20.

[10] Hallquist JO. LS-DYNA theoretical manual. Livermore Software Technology Corporation; 1998.

[11] Joldes GR, Wittek AKM. Computation of intra-operative brain shift using dynamic relaxation. Computer Methods in Applied Mechanics and Engineering 2009;198:3313-20.

[12] Rezaiee-Pajand M, Alamatian J. The dynamic relaxation method using new formulation for fictitious mass and damping. Structural Engineering and Mechanics 2010;34(1):109-33.

[13] Barnes MR. Form-finding and analysis of prestressed nets and membranes. Computers and Structures 1988;20(3):685-95.

[14] Barnes MR. Form finding and analysis of tension structures by dynamic relaxation. International Journal of Space Structures 1999;14:89-104.

[15] Ali NBH, Rhode-Barbarigos L, Smith IFC. Analysis of clustered tensegrity structures using a modified dynamic relaxation algorithm. Solids and Structures 2011(48):637-47.

[16] Underwood P. Dynamic relaxation: a review. In: Belytschko T, Hughes TJR editors. Computational methods for transient analysis, vol. 1; 1983. p. 245-65.

[17] Rio G. Herezh++: FEM software for large transformations in solids. Laboratoire d'ingénierie des matériaux de Bretagne (UEB-UBS); dépôt APP (Agence pour la Protection des Programmes)_Certification IDDN.FR.010.0106078. 000.R.P.2006.035.20600; 2011.

[18] Troufflard J, Cadou JM, Rio G. Recherche de forme des gilets de sauvetage gonflables. Mécanique et Industries 2010(11):117-22.

[19] Geuzaine C, Remacle JF. Gmsh: a three-dimensional finite element mesh generator with built-in pre- and post-processing facilities. International Journal for Numerical Methods in Engineering 2009;79(11):1309-31.

[20] Vandenbroucke A, Laurent H, Hocine N, Rio G. A hyperelasto-visco-hysteresis model for an elastomeric behavior: experimental and numerical investigations. Computational Materials Science 2010;48:495-503.

[21] Laurent H, Rio G. Formulation of a thin shell finite element with continuity c and convected material frame notion. Computational Mechanics 2001;27(3) 218-32. ISSN 0178-7675. 